\title{
PROCESOS DE TRANSPORTE EN LAS ASOCIACIONES DE OSTRÁCODOS DEL JURÁSICO INFERIOR DE LA CORDILLERA IBÉRICA, ESPAÑA
}

\author{
Carmen ARIAS \\ Department of Geological Sciences, University College London, Gower Street, \\ London WC1E 6BT, U.K. Departamento de Paleontología, F.C.C. Geológicas. \\ Universidad Complutense de Madrid. Ciudad Universitaria. 28040 Madrid.
}

\begin{abstract}
Arias, C. 1999. Procesos de transporte en las asociaciones de ostrácodos del Jurásico Inferior de la Cordillerta Ibérica, España. [Transport processes in the Lower Jurassic ostracod assemblages of the Iberian Range, Spain]. Revista Española de Paleontología, 14 (1), 1-11. ISSN 0213-6937.
\end{abstract}

\begin{abstract}
The preservational state, the relation between the number of large and small size valves (left and right valves), the population structure (the occurrence of the difference ontogenetic stages juveniles/adults) and the degree of disarticulation of carapaces (number of carapaces and valves), has been used as criteria, in some palaeoecological studies of ostracods, to know the influence of some physical processes associated with transport. For the purpose of determining the influence of this taphonomic alteration mechanism, it has been studied the Upper Pliensbachian and Lower Toarcian ostracod assemblages from eight sections situated in the Iberian Range, NE of Spain. By understanding how several taphonomic features are generated (the structure of population, relation left valve/right valve and juveniles/adults), it can be established some considerations about some palaeoenvironmental variables (rates of sedimentation, bathymetry, etc.).
\end{abstract}

Keywords: Ostracoda, taphonomy, transport, Upper Pliensbachian, Lower Toarcian, Iberian Range, Spain.

\section{RESUMEN}

El estado de conservación, el número de valvas de tamaño mayor y menor (relación entre valvas izquierdas/derechas), la estructura de la población (representación de los diferentes estadios de crecimiento juveniles/adultos) y el grado de desarticulación de los caparazones (relación entre el número de caparazones y valvas) han servido como criterios, en algunos estudios de paleoecología de ostrácodos, para conocer la influencia de algunos procesos físicos asociados con el transporte. Con el propósito de determinar la influencia de este mecanismo de alteración tafonómica, se han estudiado las asociaciones de ostrácodos de edad Pliensbachiense Superior y Toarciense Inferior en ocho secciones situadas en la Cordillera Ibérica, nordeste de España. Comprendiendo cómo se han generado varios caracteres tafonómicos (estructura de la población, relación valvas izquierdas/valvas derechas y juveniles/adultos), se pueden establecer algunas consideraciones acerca de diversas variables paleoambientales (como la tasa de sedimentación, la batimetría, etc.).

Palabras clave: Ostrácodos, tafonomía, transporte, Pliensbachiense Superior, Toarciense Inferior, Cordillera Ibérica, España.

\section{INTRODUCCIÓN}

Los análisis tafonómicos son una parte esencial en los estudios paleontológicos (Fernández López, 1981, 1984, 1985, 1986, 1988, 1989, 1991), y han sido numerosos los trabajos realizados en diferentes grupos de Invertebrados y Vertebrados. Sin embargo, ha sido sólo recientemente, cuando se ha iniciado su aplicación en algunos grupos de microfósiles, como los ostrácodos. Los estudios tafonómicos de ostrácodos son, en su mayoría, escasos, parciales y tratan, fundamentalmente, algunos aspectos relacionados con el transporte de los elementos, fenómenos de bioerosión y su empleo como índices de color en relación con el grado de maduración de la materia orgánica (Kornicker y Sohn, 1971; Langer, 1973; Kontrovitz, 1975, 1980, 1987; Kontrovitz et al., 1978, 1983, 1988, 1992; Kontrovitz y Nicholich, 1979; Kontrovitz y Snyder, 1981; Kontrovitz y Dehon, 1983; Ainsworth, et al., 1990). El objetivo de este trabajo es utilizar la información que nos aporta la composición de 


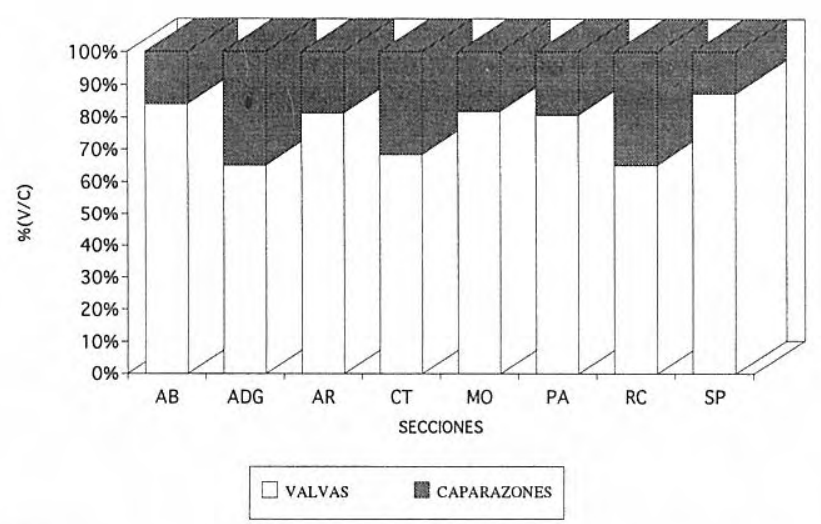

Figura 1. Porcentaje del número de valvas con relación al número de caparazones en cada sección estudiada (AB: Ablanquejo, ADG: La Almunia de Doña Godina, AR: Ariño, CT: Barranco de la Alicantas, MO: Moneva, PA: Perales de Alfambra, RC: Ricla, SP: Sierra Palomera).

las asociaciones fósiles de ostrácodos, con el propósito de conocer si han sufrido o no, algún tipo de modificación debido a procesos de transporte, y si las conclusiones extraídas nos permiten inferir características del medio y posibles condiciones de fosilización.

Los ostrácodos transportados por corrientes están sometidos a condiciones semejantes a las que afectan a cualquier partícula en un fluido (Krumbein y Sloss, 1963; Pettijohn et al., 1972). Su forma (contorno), masa, peso y tamaño influyen en su velocidad de transporte y de deposición (Allen, 1969; Kontrovitz y Nicholich, 1979; Kontrovitz y Snyder, 1981). Las diferencias en los parámetros mencionados entre las especies de ostrácodos, sus estadios de crecimiento o entre las dos valvas de su caparazón, determinan que su comportamiento sea diferente durante su transporte (Fagerstrom, 1964; Kilenyi, 1971; Breman, 1980; Whatley, 1983).

En este estudio, se han analizado las diferencias existentes en la representación de cada especie en el ámbito de sus elementos constituyentes, si se presentan articulados (caparazones) o desarticulados (valvas), y en este último caso, si predomina un tipo de valva u otro (relación entre el número de valvas de tamaño mayor y menor, ya sean las valvas izquierdas o las derechas, según las diferentes especies). Además, se ha considerado la estructura de sus poblaciones (abundancia y relación entre el número formas adultas y juveniles en cada especie).

\section{ÁREA ESTUDIADA Y METODOLOGÍA}

Se ha procedido al estudio de las asociaciones de ostrácodos extraídos de ciento trece muestras de los sedimentos de edad Pliensbachiense Superior y Toarciense Inferior de ocho cortes situados en la Cordillera Ibérica (NE de España): Ablanquejo, La Almunia de Doña Godina, Ariño, Barranco de las Alicantas, Moneva, Perales de Alfambra, Ricla y Sierra Palomera. Los materiales jurásicos de esta área han sido objeto de numerosos estudios que han incluido un detallado análisis litoestratigráfico, sedimentológico y paleontológico (ammonites, braquiópodos, foraminíferos, nanofósiles y polen) de dichos materiales (Arche et al., 1977; Comas-Rengifo, 1974, 1985; Goy, 1974, 1985; Goy et al., 1976; Arias y Comas-Rengifo, 1992; Arias et al., 1992; Herrero, 1993; Arias, 1995) y que nos han proporcionado un marco adecuado para el desarrollo de este trabajo.

La cantidad de muestra tratada osciló entre $100 \mathrm{gr}$ en el caso de margo-calizas y 300 gr en el caso de margas. El tratamiento de laboratorio incluyó el empleo de $\mathrm{NaOH}, \mathrm{H}_{2} \mathrm{O}$ y $\mathrm{H}_{2} \mathrm{O}_{2}$ para la disgregación de la muestra. Para eliminar el contenido arcilloso, se hizo pasar el sedimento a través de una columna de tamices de tamaño de luz: $0,060 \mathrm{~mm} ; 0,125 \mathrm{~mm} ; 0,250 \mathrm{~mm} ; 0,50 \mathrm{~mm}$ y 1 $\mathrm{mm}$. A continuación, se procedió a extraer todos los elementos de las cuatro fracciones de mayor tamaño.

Cada muestra fue identificada en este trabajo con un conjunto de siglas, correspondientes al nombre de la sección, y de números, que hacen referencia al nivel seleccionado. Las siglas empleadas para cada corte fueron las siguientes: AB: Ablanquejo; ADG: La Almunia de Doña Godina; AR: Ariño; CT: Barranco de las Alicantas; MO: Moneva; PA: Perales de Alfambra; RC: Ricla; SP: Sierra Palomera.

Con excepción de once muestras estériles, del resto de ellas se ha obtenido un total de 14.133 elementos que han sido asignados a 60 especies y 29 géneros de ostrácodos.

Todas las especies han sido analizadas en cada muestra y sección seleccionada, haciendo un recuento del número de caparazones y valvas, de la relación entre el número de valvas de tamaño mayor y las de tamaño menor (normalmente valvas izquierdas y derechas), y del número y relación entre formas adultas (estadio A), preadultas (A-1) y juveniles, indicando el número de elementos en cada estadio juvenil (A-2, A-3, A-4, etc.). Inicialmente, se indicarán los resultados obtenidos en cada muestra analizada y después, en la discusión, se procederá a valorar los rasgos generales que pueden extraerse del análisis.

\section{RESULTADOS}

\section{RELACIÓN ENTRE EL NÚMERO DE VALVAS Y DE CAPARAZONES}

En este estudio, se ha analizado cómo varía la proporción entre valvas y caparazones (Fig.1). En todas las secciones existe un predominio del número de valvas sobre el número de caparazones. Si consideramos la proporción de caparazones, en su conjunto, los cortes de La Almunia de Doña Godina, Barranco de las Alicantas y Ricla son los que tienen una mayor proporción de caparazones (30\%); Ariño, Moneva y Perales de Alfambra (20\%) y menor en el Ablanquejo y Sierra Palomera ( $15 \%)$.

En el corte del Ablanquejo (Fig. 2), existe un claro predominio de valvas, con excepción de las muestras, 

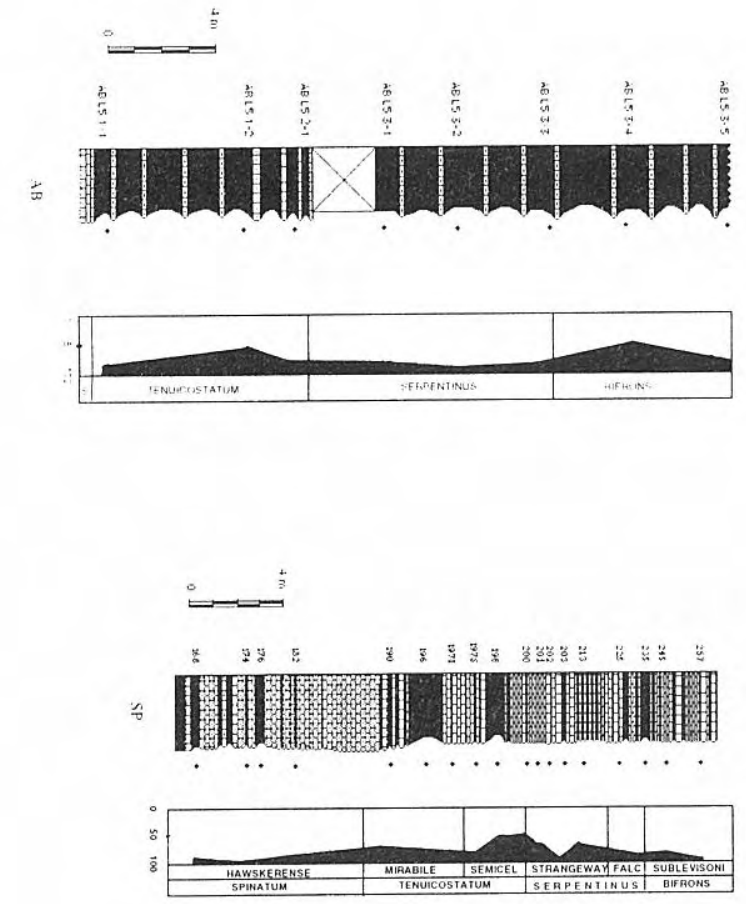

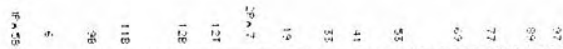
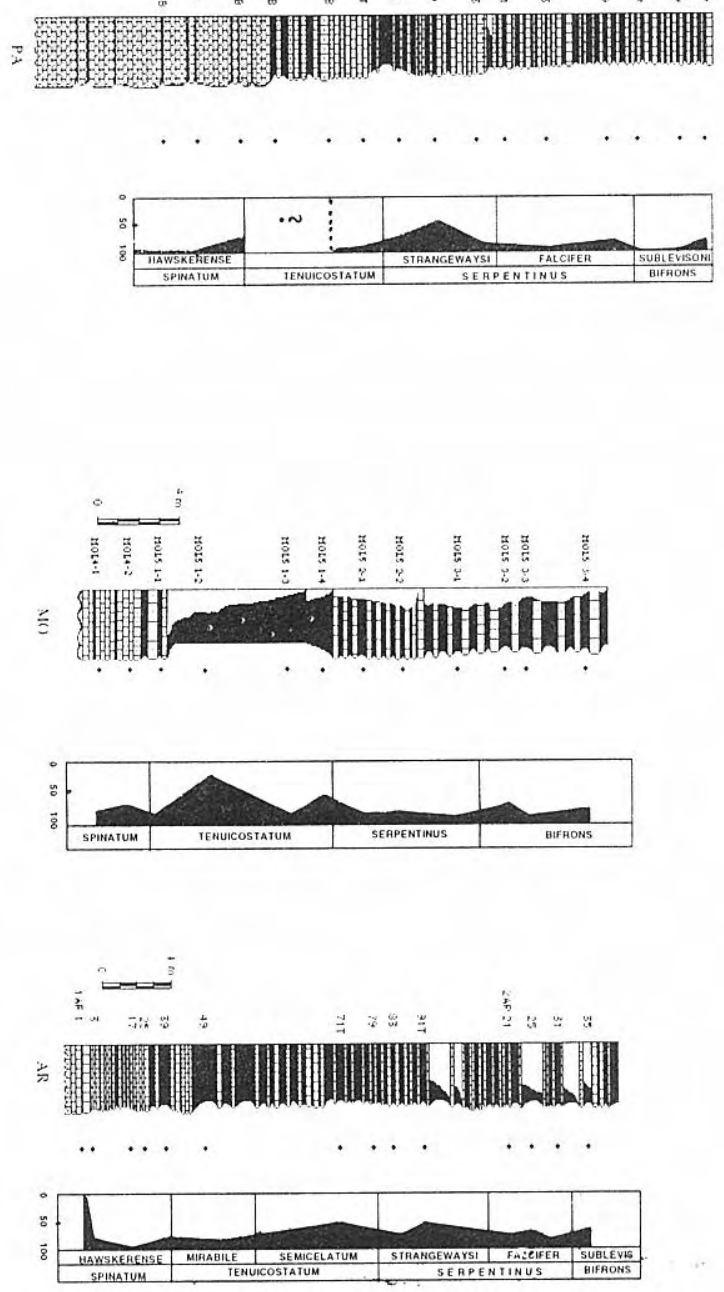
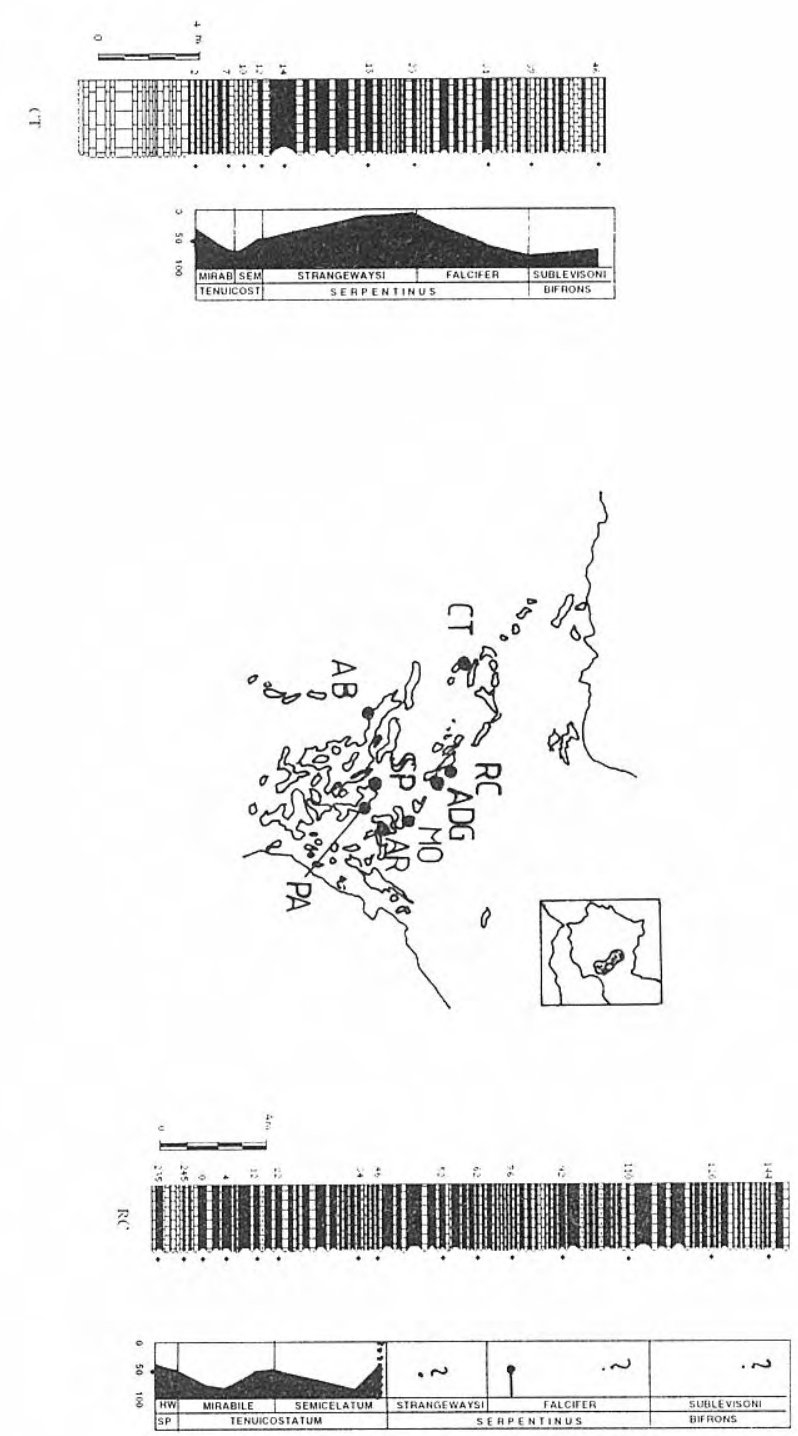

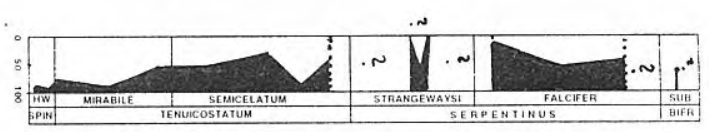

Figura 2. Variación del porcentaje del número de valvas respecto al de caparazones en cada sección y muestra estudiada. Siglas como en la Fig. 1. En negro, porcentaje de caparazones. El signo de interrogación indica que no se han encontrado ejemplares en las muestras. 
AB.L. $L_{5}$ 1-2 de la Zona Tenuicostatum y AB.L. L $_{5}$-4 de la Zona Bifrons, Subzona Sublevisoni. En la sección de La Almunia de Doña Godina (Fig. 2), predominan las valvas sobre los caparazones, excepto en las muestras ADG.34 de la Subzona Mirabile y ADG.76 de la Subzona Falcifer. En el corte de Ariño (Fig. 2), en todas las muestras predominan las valvas frente a los caparazones, con excepción de la muestra 1AR.1 de la Zona Spinatum, Subzona Hawskerense y la muestra 1AR.71T de la Zona Tenuicostatum, Subzona Semicelatum. En la sección del Barranco de las Alicantas (Fig. 2), la proporción de caparazones es muy alta en la mitad de las muestras, particularmente, en las muestras 1CT.18 y 1CT.23 de la Subzona Strangewaysi. En la sección de Moneva (Fig. 2), predominan las valvas en la mayoría de las muestras estudiadas, excepto en MO.L . $_{5}$-1-2 y MO.L. L $_{5}$.1-4 de la Zona Tenuicostatum. En el corte de Perales de Alfambra (Fig. 2), dominan las valvas, excepto en la muestra 2PA.19 de la Subzona Strangewaysi, Zona Serpentinus. Del mismo modo, en el corte de Ricla (Fig. 2), predominan las valvas, con un elevado número de caparazones en las muestras RC.12 y RC.46 de la Zona Tenuicostatum. En la sección de Sierra Palomera (Fig. 2), las valvas son más numerosas que los caparazones en todas las muestras, con un máximo en el número de caparazones en la muestra de SP.198 de la Zona Tenuicostatum, Subzona Semicelatum.

RELACIÓN ENTRE EL NÚMERO DE VALVAS DE TAMAÑO MAYOR Y LAS DE TAMAÑO MENOR (NÚMERO DE VALVAS IZQUIERDAS Y DE VALVAS DERECHAS)

Otro aspecto considerado en muchos trabajos ha sido la proporción entre el número de valvas de tamaño mayor y las de tamaño menor. La mayoría de las especies de ostrácodos estudiadas en este trabajo se caracterizan por poseer dos valvas de diferente tamaño (generalmente la valva izquierda es la de tamaño mayor) y contorno, cuyas diferencias varían según las especies y aumentan desde las primeras formas larvales al estadio adulto.

En nuestro trabajo, las diferencias más importantes entre ambas valvas (en forma y tamaño) se observan en los ejemplares de las especies de los géneros Ogmoconchella Gründel, Cytherella Jones, Gramannella Lord, Ektyphocythere Bate, Kinkelinella Martin y Praeschuleridea Bate. En los restantes géneros, la principal diferencia entre las valvas es el tamaño. Estas diferencias marcan un distinto comportamiento durante el transporte de las valvas y la separación entre las valvas izquierdas y derechas (Pokorny, 1978). Aunque la valva izquierda es, comúnmente, la de tamaño mayor, las dos especies del género Cytherella presentes en este estudio se caracterizan por tener la valva derecha como valva de tamaño mayor. Por este hecho, y aunque el índice utilizado generalmente en este tipo de análisis ha sido la relación VI/VD, en este estudio se ha considerado preferible referirse a la relación entre el número de valvas de tamaño mayor/valvas de tamaño menor.
Si analizamos la proporción entre el número de valvas de tamaño mayor y las de tamaño menor en todas las secciones (Fig.3), se observa que en las secciones del Ablanquejo, Ariño, Moneva, Ricla y Sierra Palomera existe un predominio de las valvas de tamaño mayor respecto a las de tamaño menor, mientras en la Almunia, Barranco de las Alicantas y Perales de Alfambra se da la situación opuesta.

En todas las muestras estudiadas en las secciones del Ablanquejo y Ricla, predominan las valvas de tamaño mayor respecto a las de tamaño menor. En la sección de Ariño, sólo en la muestra 1AR.71T de parte superior de la Zona Tenuicostatum, Subzona Semicelatum se ha registrado un número mayor de valvas de tamaño menor. En la sección de Moneva esta situación se ha registrado en las muestras MO. $\mathrm{L}_{4} \cdot 1-2$ de la parte superior de la Zona Spinatum, MO.L. $L_{5} \cdot 1-1$ de la parte inferior de la Zona Tenuicostatum, MO.L. . $_{5}$ - 4 de la parte superior de la Zona Tenuicostatum, MO.L. . $_{5}-1$ y MO.L. L $_{5}$-2-2 de la parte inferior de la Zona Serpentinus. En Sierra Palomera, el número de valvas de tamaño menor es superior al de valvas de tamaño mayor en las muestras SP.174 y SP.182 de la Zona Spinatum; SP.196 de la Zona Tenuicostatum, Subzona Mirabile; SP.198 de la Zona Tenuicostatum, Subzona Semicelatum; SP.201 y SP.202 de la parte inferior de la Zona Serpentinus, Subzona Strangewaysi y SP.257 de la Zona Bifrons.

El número de valvas de tamaño menor es más elevado que el número de valvas de tamaño mayor en todas las muestras de la sección del Barranco de las Alicantas excepto en la muestra 1CT.31 de la parte superior de la Zona Serpentinus, Subzona Falcifer y en 1CT.37 de la parte inferior de la Zona Bifrons. En la Almunia de la Doña Godina, el número de valvas de tamaño mayor es superior en la muestra ADG.2 de la Zona Spinatum, ADG.40 de la Zona Tenuicostatum, Subzona Semicelatum, ADG66.B de la Zona Serpentinus, Subzona Strangewaysi y ADG.76 de la Zona Serpentinus, parte inferior de la Subzona Falcifer. En la sección de Perales de Alfambra, el dominio de las valvas de tamaño menor no se cumple en las muestras 1.PA.6 de la Zona Spinatum; 1PA.9B, 1PA.12B y 1PA.12T de la parte superior de la Zona Tenuicostatum; 2PA.19 de la Zona Serpentinus, Subzona Strangewaysi y 2PA.77 de la parte inferior de la Zona Bifrons.

Si consideramos de una manera global los resultados obtenidos, se pueden ver ciertas tendencias generales en el modo de variar la relación entre ambas valvas. En las muestras de edad Pliensbachiense Superior predominan las valvas de tamaño mayor, en el límite Pliensbachiense Superior-Toarciense se equilibra el número entre ambas valvas y en el inicio del Toarciense (base de la Zona Tenuicostatum) predominan de nuevo las valvas de mayor tamaño. En la parte superior de la Zona Tenuicostatum la representación se equilibra otra vez, y en el inicio de la Zona Serpentinus, se inicia un predominio de las valvas de tamaño menor. En el resto de la Zona Serpentinus y en la parte inferior de la Zona Bifrons, la relación se invierte y existe de nuevo un predominio de las valvas de tamaño mayor. 
市
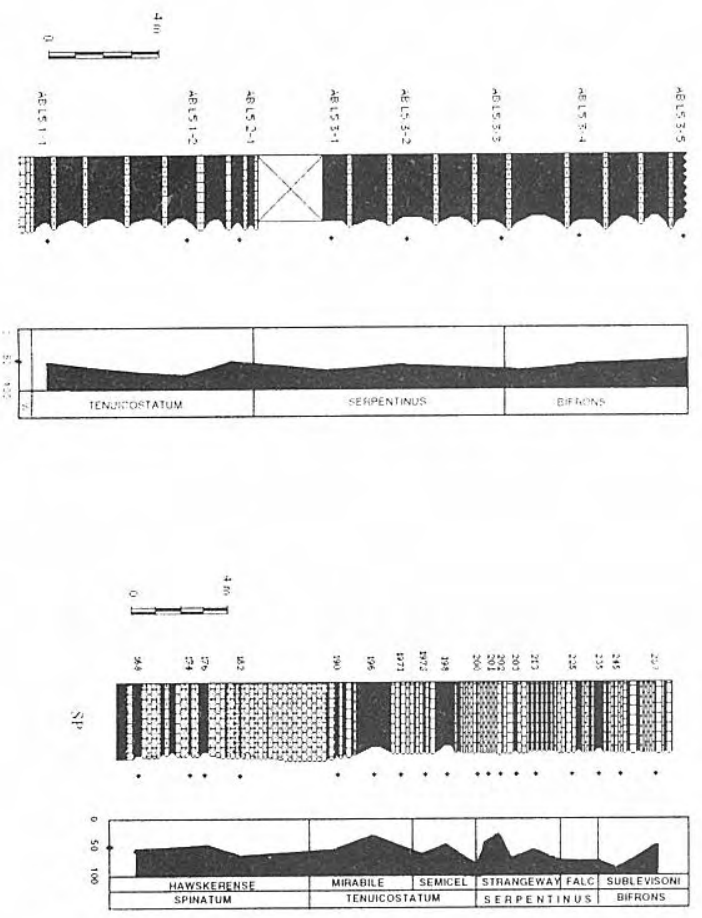

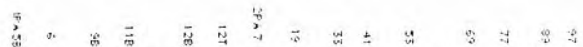
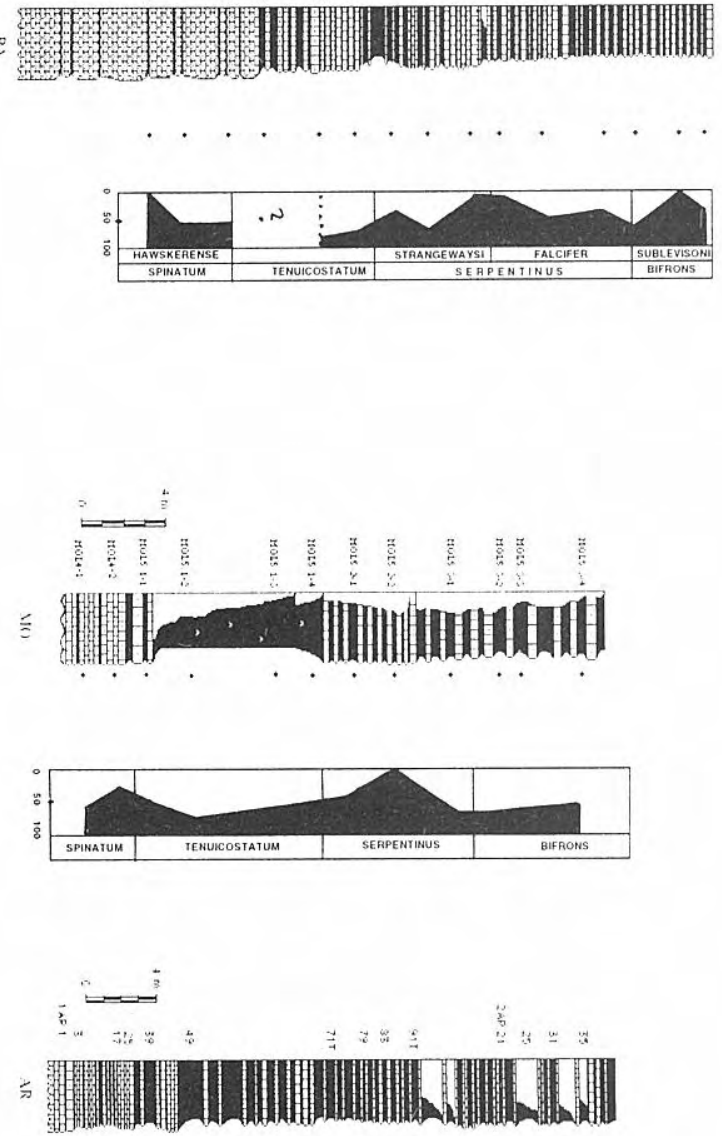

.. $\quad .$.

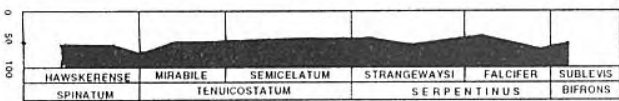

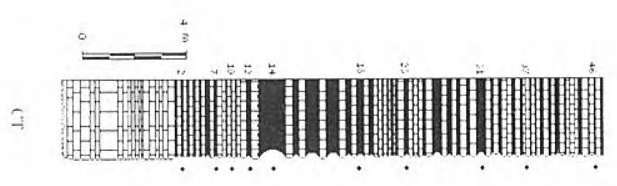
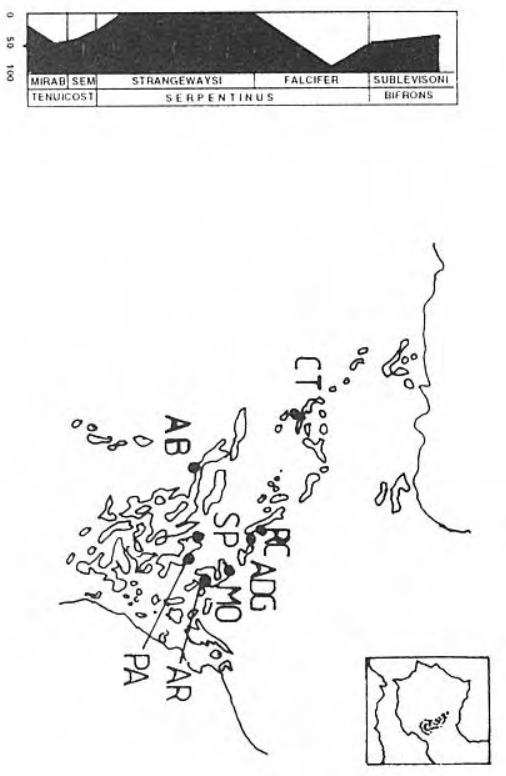

곤
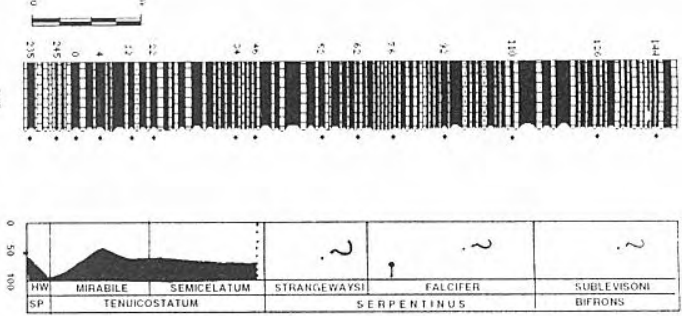

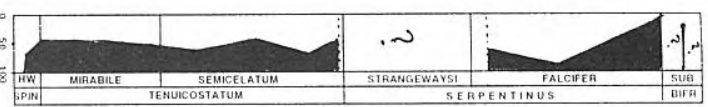

Figura 3.Variación del porcentaje de la relación entre el número de valvas de tamaño mayor y tamaño menor en cada sección y muestra estudiada. Siglas como en la Fig. 1. En negro, porcentaje de valvas de tamaño menor. El signo de interrogación indica que no se han encontrado ejemplares en las muestras. 


\section{ESTRUCTURA DE LAS POBLACIONES FÓSILES}

El análisis de la estructura de las poblaciones en las asociaciones fósiles de ostrácodos necesita de la presencia de un material rico, con poblaciones de especies bien representadas, donde se hayan registrado un número elevado de elementos por cada estadio de crecimiento. Es muy difícil que podamos tener todas las mudas correspondientes a la ontogenia completa de los individuos de ostrácodos, a no ser que se trabaje con poblaciones actuales bajo unas condiciones especiales, como sería el caso de experimentos realizados en un tanque, donde no se producen pérdidas por transporte o procesos diagenéticos.

En nuestro estudio son pocas las especies que cuentan con una buena representación de todos de los estadios de crecimiento. Aunque extraer consecuencias generales con este tipo de análisis es muy arriesgado, hemos resumido los datos del estudio, indicando los resultados por cada muestra analizada (Fig. 4).

Como primera aproximación, debemos indicar que ninguna especie está representada en todos los cortes del mismo modo, en unos cortes presenta un registro más completo que en otros. Sin embargo, sí se ha detectado que las especies de healdiidos y cyprideos tienen una representación más completa en el corte de Ariño, los cytherellideos, en el corte del Ablanquejo y los cytheráceos, en el corte de Moneva.

Si analizamos cada corte, observamos que la sección de Ariño es la que contiene un mayor número de especies bien representadas en todos sus estadios de crecimiento, (adultos y un número importante de estadios larvales). Peor es la representación en las secciones del Ablanquejo y Moneva, y en el corte del Barranco de las Alicantas. En las secciones de La Almunia de Doña Godina, Perales de Alfambra, Ricla y Sierra Palomera, el número de elementos registrado es bastante bajo en todas las especies, y por tanto, la representación en cada especie de sus estadios de crecimiento es muy pobre.

Si estudiamos como varía por secciones, analizando todas las especies presentes en cada una de ellas, la estructura de las asociaciones de ostrácodos registradas observamos que durante el Pliensbachiense Superior, en las secciones de Ariño y Moneva, la mayoría de las especies descritas se caracterizan por estar bien representadas (estando presentes sus formas adultas, así como, un gran número de formas larvales). En las secciones de Sierra Palomera y La Almunia de Doña Godina, las especies se caracterizan por no tener una buena representación de sus estadios adultos (Fig. 4).

Durante la Zona Tenuicostatum, en los cortes de Ariño y Barranco de las Alicantas, las especies muestran una buena representación de sus estadios juveniles de mayor tamaño (A-1). En los cortes del Ablanquejo, Moneva y Ricla, las especies están bien representadas en todos sus estadios de crecimiento, excepto durante el tránsito entre las subzonas Mirabile y Semicelatum, donde existe una representación pobre de los estadios adultos. En el resto de la Zona Tenuicostatum, y parte inferior de la Subzona Strangewaysi, en los cortes del
Ablanquejo, Moneva y Ricla, vuelven a estar bien representados todos los estadios incluidos los adultos (Fig. 4).

En la parte superior de la Subzona Strangewaysi, cambia de nuevo la estructura, y no están presentes los adultos en los cortes de Ricla y Sierra Palomera, ni la mayoría de los estadios juveniles en los cortes de Moneva y Perales de Alfambra. Durante la Subzona Falcifer, en los cortes del Ablanquejo, Ariño, Barranco de las Alicantas, Moneva y Sierra Palomera, las poblaciones presentan series ontogénicas casi completas, faltan las formas adultas en el corte de Perales de Alfambra y predominan las formas larvales en la Almunia de Doña Godina y las adultas en el Barranco de las Alicantas (Fig. 4).

En el inicio de la Zona Bifrons, en los cortes del Ablanquejo, la Almunia de Doña Godina, Barranco de las Alicantas y Sierra Palomera, todos los estadios están bien representados, faltando los ejemplares adultos en los cortes de Moneva y Perales de Alfambra. En la parte superior de la Subzona Sublevisoni, Zona Bifrons, están bien representados todos los estadios en los cortes del Ablanquejo y Moneva, y faltan los ejemplares adultos de los cortes de Perales de Alfambra y Sierra Palomera (Fig. $4)$.

\section{DISCUSIÓN}

\section{NÚMERO DE VALVAS Y DE CAPARAZONES}

La relación entre el número de caparazones y valvas es un índice muy empleado en Paleoecología de las asociaciones de ostrácodos (Kilenyi, 1969, 1971; Pokorny, 1971, 1978; Oertli, 1971; Michelsen, 1975, Breman, 1980). Existen diversos factores que determinan el grado de desarticulación de las formas, pero, principalmente, se deben considerar sus características anatómicas (tipo de charnela, ligamentos, solapamiento, etc.) y las características del medio (duración e intensidad del transporte, tasa de sedimentación y energía del medio).

La desarticulación del caparazón se produce inmediatamente después de la muerte del organismo y durante los procesos de ecdisis (Elofson, 1941; Langer, 1973; Pokorny, 1978). Al morir el animal, las valvas se abren por la acción bacteriana. Pero si la tasa de sedimentación es alta y las condiciones energéticas del medio son moderadas o bajas, puede producirse el enterramiento de los caparazones y, de este modo, se dificulta su desarticulación (Pokorny, 1971; Kidwell y Jablonski, 1983; Parsons y Brett, 1991; Speyer y Brett, 1991).

Esta relación está influenciada, también, por los procesos de transporte, que va a suponer una selección de los elementos en función de su forma, tamaño, volumen, etc. Así, la forma hidrodinámica de cada especie, y su representación como formas articuladas o desarticuladas, influye en el modo en que sus elementos son transportados, produciéndose asociaciones con morfologías semejantes, que indicarían una misma 


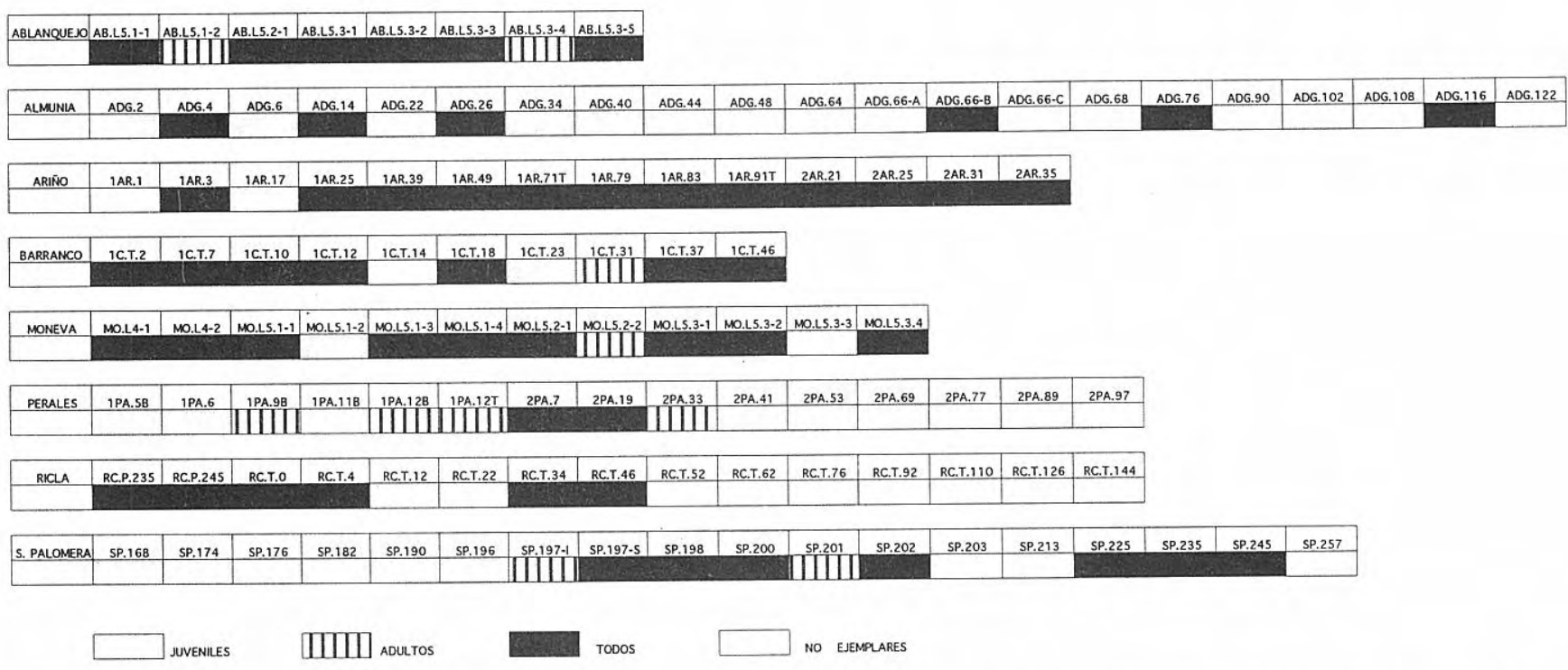

Figura 4. Composición de las asociaciones de ostrácodos en cada muestra de las secciones estudiadas, indicando si están integradas únicamente por elementos juveniles, adultos o bien comprenden ambos tipos.

respuesta frente a la acción de las corrientes hidráulicas. Así, los caparazones, al ser biconvexos, se apoyan sobre una superficie convexa, mientras las valvas lo hacen con el lado convexo hacia arriba (apoyándose sobre su margen externo) con lo que se necesita el doble de velocidad para que comiencen a moverse (Kontrovitz, 1975; Kontrovitz et al., 1978, Adamczak, 1982). Kontrovitz y Snyder (1981) calcularon que la velocidad necesaria para los caparazones era de $16,3 \mathrm{~cm} / \mathrm{s}$ y para las valvas de $21,4 \mathrm{~cm} / \mathrm{s}$. Además, y dado que los caparazones tienen algo menos del doble del volumen que el que representarían las dos valvas, su velocidad de tracción media es también menor (Kontrovitz et al., 1978; Kontrovitz y Nicholich, 1979).

Como ya ha sido indicado, los resultados de nuestro trabajo muestran un predominio del número de valvas en todas las secciones (Fig. 1), lo que podría indicar una energía y una tasa de sedimentación moderadas en el medio. La energía sería suficiente para desplazar los caparazones (que habrían sido introducidos en el medio tras su transporte), pero no las valvas que necesitan mayor energía para ser transportadas.

Para estudiar la influencia de los factores ambientales, debemos considerar si el mayor número de caparazones en algunas muestras (más de un tercio) se debe a las propias características morfológicas y anatómicas del organismo, particularmente, su mecanismo de articulación (tipo de charnela, ligamento, solapamiento, tejido, etc.). Para ello debemos establecer si existe una tendencia determinada, en cada especie, a presentarse como formas articuladas, desarticuladas o bien alternan dicha tendencia según las secciones analizadas.

En el corte del Ablanquejo, existe un claro predominio de las valvas sobre los caparazones, con excepción de dos muestras, caracterizadas por un alto número de caparazones de Liasina lanceolata Apostolescu, Bairdiacypris dorisae (Knitter) y Pseudomacrocypris subaequalis Michelsen. En la sección de La Almunia de Doña Godina, predominan las valvas sobre los caparazones, con excepción de dos muestras, donde se ha detectado una alta proporción de caparazones de Liasina lanceolata y Pseudomacrocypris subtriangularis Michelsen. En el corte de Ariño, en todas las muestras predominan las valvas frente a los caparazones, excepto en una muestra debido a la elevada representación de caparazones de Liasina lanceolata. En la sección del Barranco de las Alicantas, la proporción de caparazones es muy alta en dos muestras, donde dominan los ejemplares articulados de Bairdiacypris dorisae. La elevada proporción de las valvas registrada en el corte de Moneva se reduce en dos muestras, como consecuencia del número alto de caparazones de Cytherella toarcensis Bizon y Liasina lanceolata. Del mismo modo, en el corte de Perales de Alfambra, el predominio de caparazones en una muestra es debido al alto número de caparazones de Cytherella toarcensis y Liasina lanceolata. En el corte de Ricla, el predominio de caparazones es muy alto en dos muestras, que se caracterizan por un alto porcentaje de caparazones de Liasina lanceolata. En la sección de Sierra Palomera, en la muestra en donde se alcanza el mayor porcentaje de caparazones es consecuencia del número importante de caparazones de Cytherella toarcensis.

Aunque la mayoría de las especies de este estudio no muestran una tendencia fija a presentarse como formas articuladas o desarticuladas, las especies antes citadas (excepto Cytherella toarcensis y Liasina lanceolata), se presentan, casi siempre y en su totalidad, como formas articuladas, y son las responsables del alto número de caparazones registrados en algunas de las muestras. Por la continuidad como formas articuladas de estas especies, en las distintas muestras y cortes, podemos pensar que es una consecuencia debida a su mecanismo de articulación. Las especies citadas tienen en su mayoría, una charnela de tipo adonta (Bairdiacypris, Cytherella, Cytherelloidea, Isobythocypris, Liasina, Monoceratina, Paracypris y 
Monoceratina) y, en menor proporción, merodonta (Pseudomacrocypris y Monoceratina). Posiblemente, además del tipo de charnela, el tipo de solapamiento o las características de los músculos abductores, son la causa de que estas especies muestren un alto porcentaje (generalmente más de un 90\%) de caparazones, en todas las muestras de este trabajo.

Si consideramos como primera aproximación, que las especies citadas tienden a presentarse como formas articuladas debido a sus características morfológicas, independientemente de los factores ambientales, únicamente dos especies, Liasina lanceolata y Cytherella toarcensis pueden facilitarnos información sobre las diferentes condiciones ambientales, puesto que alternan su presencia como formas articuladas y desarticuladas, según las muestras o secciones analizadas. Liasina lanceolata se presenta, normalmente, desarticulada en todas las muestras, con excepción de las muestras de la parte superior de la Zona Tenuicostatum de las secciones del Ablanquejo, Ariño, Moneva, Ricla y Sierra Palomera; y Cytherella toarcensis presenta un comportamiento semejante, detectándose la mayor proporción de caparazones en la parte superior de la Zona Tenuicostatum de los cortes del Ablanquejo, La Almunia de Doña Godina, Ariño, Moneva y Sierra Palomera. De este modo, ambas especies tienden a presentarse como formas articuladas, únicamente en la parte superior de la Zona Tenuicostatum, mientras que predominan como formas desarticuladas, en el resto de las zonas.

Si analizamos la relación v/c en su conjunto, se observa que varía entre las secciones analizadas (Figs. 1, 2). En la Almunia de Doña Godina, Barranco de las Alicantas y Ricla, es más alta que en Ariño, Perales de Alfambra y Moneva, y en estos últimos, más que en el Ablanquejo y Sierra Palomera. Según estos datos, en la sección del Barranco de las Alicantas y, fundamentalmente, de la Almunia de Doña Godina y Ricla, podrían haber existido condiciones de energía menor que en los otros cortes, aumentando la energía progresivamente en las secciones de Ariño, Moneva y Perales de Alfambra, donde la proporción de valvas es mayor, desarrollándose las condiciones más energéticas en los cortes de Sierra Palomera y el Ablanquejo.

Otro factor determinante podría ser la cantidad original de oxígeno del medio. Así, además del desarrollo de determinadas condiciones energéticas, podría existir una disminución en la proporción de oxígeno, que inhibiría en parte la acción de las bacterias aeróbias, que afectarían a la abertura de los caparazones, suponiendo un posible desarrollo de condiciones más anóxicas desde los cortes de Sierra Palomera, Ablanquejo, Ariño, Moneva, Perales de Alfambra, a los del Barranco de las Alicantas, la Almunia de Doña Godina y Ricla.

NÚMERO DE VALVAS DE TAMAÑO MAYOR/ MENOR (NUMERO DE VALVAS IZQUIERDAS/ DERECHAS)

Si consideramos las variaciones en el número de valvas de tamaño mayor respecto a las de tamaño menor, se observa que, en la mayoría de las muestras, existe un predominio más o menos marcado de las valvas de tamaño mayor, excepto en la parte superior de la Zona Tenuicostatum de los cortes de Ariño, la Almunia de Doña Godina, Barranco de las Alicantas, Moneva y Sierra Palomera; y en la parte inferior de la Zona Serpentinus de los cortes de la Almunia de Doña Godina, Perales de Alfambra, Moneva y Sierra Palomera, donde las valvas de menor tamaño predominan.

Este hecho podría indicar que, durante el intervalo temporal estudiado, parecen haber existido condiciones de energía relativamente alta, que permitieron el transporte de las valvas de tamaño más pequeño, pero también, aunque en menor medida, de las mayores. Sin embargo, en la parte superior de la Zona Tenuicostatum e inferior de la Zona Serpentinus, la energía pudo disminuir (Fig. 3) y sólo las valvas más pequeñas pudieron ser transportadas.

\section{ESTRUCTURA Y COMPOSICIÓN DE LAS POBLACIONES FÓSILES DE OSTRÁCODOS}

Los procesos de transporte afectan también a la proporción de formas adultas y juveniles. Las diferencias de tamaño, forma, densidad, peso, etc., entre los estadios de crecimiento van a dar lugar a un proceso de selección por la acción de las corrientes de fondo. Cuando se estudia la estructura de las poblaciones de los ostrácodos, se debe tener en cuenta que los primeros estadios larvales están representados por formas de tamaño muy pequeño y frágiles, lo que hace muy difícil su conservación, excepto en condiciones especiales. Los estudios sobre ostrácodos actuales nos han permitido conocer que además de sus características morfológicas, estructura de sus valvas, o mineralogía, influyen también determinados condicionamientos (fenómenos de mortalidad infantil o etológicos, como la segregación entre adultos/juveniles, etc.) o ecológicos (cambios en los parámetros ambientales, como la salinidad, temperatura, etc.). Así pues, la presencia de numerosos ejemplares juveniles podría o no indicar la actuación de determinados mecanismos de alteración tafonómica (selección hidráulica por transporte, abrasión, diagénesis) y esta relación debe utilizarse con precaución.

La composición de las poblaciones va a estar influida por la energía del medio. En medios de baja energía, la estructura de la población comprende adultos de ambos. sexos y un gran número de juveniles, que no muestran rasgos tafonómicos que indiquen que hayan sufrido procesos de transporte. En medios de alta energía, la estructura de la población está formada por adultos, algunas mudas de los estadios penúltimos, y no hay juveniles al haber sido removidos por corrientes u otros mecanismos de transporte (Whatley, 1988). Este autor desarrolló un modelo hipotético, basado en el estudio de numerosas poblaciones actuales, en el que existiría una distribución de los elementos hacia el mar, de modo que un aumento en la profundidad significaría la presencia de una proporción mayor de ejemplares juveniles, que cada vez serían de menor tamaño. En las áreas marginales o costeras, tendríamos poblaciones con adultos de ambos 
sexos y representantes del último estadio larval (A-1, preadulto), después al aumentar la profundidad, predominarían representantes del estadio A-1 y A-2 (estadio larval previo al A-1), después, de A-2 y A-3, y así sucesivamente. Es decir, existiría una distribución ideal de los distintos estadios, disminuyendo su tamaño desde la línea de costa hacia las zonas más profundas, coincidiendo con la dirección del transporte de los sedimentos (lo que supone una disminución progresiva del tamaño de grano transportado).

En el presente trabajo, se ha podido apreciar una variación en la estructura de las poblaciones fósiles entre las distintas secciones, desde las registradas en el corte de Ariño (las más abundantes y completas) a las descritas en las secciones de Ablanquejo, Moneva y Barranco de las Alicantas (algo menos abundantes e incompletas), hasta aquellas descritas en las secciones de La Almunia de Doña Godina, Perales de Alfambra, Ricla y Sierra Palomera (muy incompletas) (Fig. 4). Además, se detectan cambios importantes en todas las especies de las muestras del tránsito entre las subzonas Mirabile y Semicelatum de la Zona Tenuicostatum; y entre las subzonas Strangewaysi y Falcifer de la Zona Serpentinus (Fig. 4). Si consideramos este modelo en nuestro trabajo, las muestras de edad Pliensbachiense Superior podrían indicar zonas más próximas a la línea de costa, mientras las muestras de la Zona Tenuicostatum representarían zonas más profundas.

Más difícil es extraer este tipo de conclusiones para cada sección, pues para este estudio no hemos considerado otros aspectos de alteración tafonómica que influirían decisivamente en el grado de conservación de los elementos, y porque la riqueza de representación de las poblaciones fósiles varía según la sección estudiada. Tenemos secciones como el Ablanquejo, Ariño o Moneva, con asociaciones de ostrácodos especialmente ricas, frente a otras, como La Almunia de Doña Godina y Perales de Alfambra, donde las asociaciones son muy pobres. Sin embargo, en una aproximación a los resultados obtenidos en este trabajo, se podría decir, que en todas las secciones estudiadas las asociaciones de ostrácodos nos indican la existencia de un ambiente relativamente somero y de baja energía, con un aumento de la profundidad, más patente en las secciones de la Almunia de Doña Godina y Sierra Palomera, al inicio del Toarciense, y en las secciones del Ablanquejo', la Almunia de Doña Godina, Moneva, Sierra Palomera y Ricla, durante el tránsito entre las subzonas Mirabile y Semicelatum de la Zona Tenuicostatum.

\section{CONCLUSIONES}

El análisis de las estructuras de las poblaciones y el estado de conservación de los elementos registrados indican cómo la acción mecánica de los procesos de transporte debió ser muy importante en nuestra área de estudio. Los resultados parecen señalar que con excepción del intervalo que incluye la parte superior de la Zona Tenuicostatum e inferior de la Zona Serpentinus, donde debieron existir condiciones más bajas de energía, la energía del medio fue moderada.

Las características observadas en los elementos registrados y en las asociaciones parecen indicar la existencia de condiciones más estables en el medio, que, además, sería posiblemente pobremente oxigenado, en las secciones de Perales de Alfambra, Barranco de las Alicantas, la Almunia de Doña Godina y Ricla, con respecto a Ariño, Moneva, Sierra Palomera y el Ablanquejo.

Si hacemos una estimación general de todos los datos observados, los resultados parecen indicar un aumento en la profundidad del medio, que se iniciaría próximo al límite entre las zonas Spinatum y Tenuicostatum, y que progresaría durante el Toarciense inferior.

\section{AGRADECIMIENTOS}

Quisiera agradecer al Dr. Sixto Fernández López (Universidad Complutense de Madrid) y a la Dra. María Luisa Martínez Chacón (Universidad de Oviedo) por su inestimable ayuda en la corrección de este manuscrito. Además, quisiera dar las gracias a la Dra. María José Comas-Rengifo y a la Dra. Concha Herrero (Universidad Complutense de Madrid), al Dr. Guillermo Meléndez (Universidad de Zaragoza) y al Dr. Luis Carlos Sánchez de Posada (Universidad de Oviedo) por la revisión del manuscrito, y a todas aquellas personas que actuando como revisores, han tenido la amabilidad de leerlo, corregir muchos errores, y por sus útiles sugerencias, que mejoraron y ayudaron a terminar este trabajo. Este estudio fue realizado dentro del Proyecto PB93-0459 de la DGICYT (España) y fue terminado gracias a una beca de la Unión Europea del Programa Training and Mobility of Researchers Programme (TMR Programm-ERBFMBICT95-0544).

\section{BIBLIOGRAFÍA}

Adamczack, F.J. 1982. Preliminary results of a study of sedimentary structures and orientation of ostracode valves in carbonate rocks of Gotland, Sweden. Stockholm Contributions in Geology, 37, 1-8.

Ainsworth, N.R., Burnett, D.B. and Kontrovitz, M. 1990. Ostracod colour change by thermal alteration offshore Ireland and Western UK. Marine and Petroleum Geology, 7, 288-297.

Allen, J.R. 1969. The maximum slope angle attainable by surfaces underlain by bulked equal spheroids with variable dimensional ordering. Geological Society of America Bulletin, 80, 1923-1930.

Arche, A., Comas-Rengifo, M.J., Gómez, J.J. y Goy, A. 1977. Evolución vertical de los sedimentos carbonatados del Lías medio y superior en Sierra Palomera (Teruel). Estudios Geológicos, 33, 571-574.

Arias, C. 1995. Los ostrácodos del Domeriense superior y Toarciense inferior de la Cordillera Ibérica. Tesis Doctoral. Fac C.C. Geológicas, Universidad Complutense de Madrid, 521 pp. (Inédita). 
Arias, C. y Comas-Rengifo, M.J. 1992. Ostrácodos del Domeriense superior y Toarciense inferior de la Cordillera Ibérica. Revista Española de Micropaleontología, 3, 111-135.

Arias, C., Comas-Rengifo, M.J., Goy, A., Herrero, C. et Ruget, Ch. 1992. Variations dans les associations de brachiopodes, foraminifères et ostracodes du Toarcien basal dans le secteur central de la Cordillère Ibérique: un exemple dans La Rambla del Salto (Teruel, Espagne). Cahiers de l'Institut Catholique de Lyon, 5, 5-25.

Breman, E. 1980. Differential distribution of left and right ostracode valves in the Adriatic Sea. Palaeogeography, Palaeoclimatolology, Palaeoecolology, 32, 135-141.

Comas-Rengifo, M.J. 1974. Estudio paleontológico y bioestratigráfico del Jurásico de Ribarredonda (Guadalajara). Tesis de Licenciatura, Facultad de Ciencias Geológicas U.C.M. Madrid (Inédita).

Comas-Rengifo, M.J. 1985. El Pliensbachiense de la Cordillera Ibérica. Tesis Doctoral, Facultad de Ciencias Geológicas U.C.M. Madrid, 591 pp. (Inédita).

Elofson, O. 1941. Zur Kenntnis der marinen Ostrakoden Schwedens mit besonderer Berücksichtigung des Skagerraks. Zoologiska Bidrag fran Uppsala, 19, 215253.

Fagerstrom, J.A. 1964. Fossil communities in paleoecology: their recognition and significance. Bulletin of the Geological Society of America, 75, 1197-1216.

Fernández López, S., 1981. La evolución tafonómica (un planteamiento neodarwinista). Boletín de la Real Sociedad Española de Historia Natural, 79, 243-254.

Fernández López, S. 1984. Nuevas perspectivas de la Tafonomía evolutiva: tafosistemas y asociaciones conservadas. Estudios Geológicos, 40, 215-224.

Fernández López, S. 1985. Criterios elementales de reelaboración tafonómica en ammonites de la Cordillera Ibérica. Acta Geológica Hispánica, 19, 29-45.

Fernández López, S. 1986. Sucesiones paleobiológicas y sucesiones registráticas (nuevos conceptos paleontológicos). Revista Española de Paleontología, 1, 29-45.

Fernández López, S. 1988. La Tafonomía: un subsistema conceptual de Paleontología. Coloquios de Paleontología, 41, 9-34.

Fernández López, S. 1989. La materia fósil. Una concepción dinamicista de los fósiles. In: Nuevas tendencias en Paleontología (Ed. E. Aguirre). Consejo Superior de Investigaciones Científicas, Madrid, 25-45.

Fernández López, S. 1991. Taphonomic concepts for a theoretical biochronology. Revista Española de Paleontología, 6, 37-49.

Goy, A. 1974. El Lías de la unidad norte de la Rama Castellana de la Cordillera Ibérica. Tesis Doctoral, Facultad de Ciencias Geológicas, U.C.M. Madrid, 3, 940 pp.

Goy, A., 1985. Jurassique des Ibérides. Actes du Laboratoire du Géologie, Sédimentologie et Paléontologie de l'Université Paul Savatier (Extr. Guide de excursions), 2 (2), 32-56.

Goy, A., Gómez, J.J. y Yébenes, Y. 1976. El Jurásico de la Rama Castellana de la Cordillera Ibérica (mitad norte). I. Unidades Litoestratigráficas. Estudios Geológicos, 32, 391-423.
Herrero, C. 1993. Los Foraminíferos del Toarciense inferior de la Cordillera Iberica. Colección Tesis Doctorales, 87/93, Ed. Universidad Complutense, 523 pp.

Kidwell, S.M. and Jablonsky, D. 1983. Taphonomic Feedback. Geological Consequences of Shell Accumulation. In: Biotic Interactions in recent and fossil benthic communities (Eds. M.J.S. Tevesz y P.L. McCall), Plenum Press, 5, 195-248.

Kilenyi, T.I. 1969. The problems of ostracod ecology on the Thames Estuary. In: The Taxonomy, Morphology and Ecology of Recent Ostracoda (Ed. J.W. Neale). Oliver and Boyd, Edinburgh, 251-267.

Kilenyi, T.I. 1971. Some basic questions in the paleoecology of ostracods. Colloque sur la Paléoécologie des Ostracodes (Ed. H.J. Oertli). Bulletin du Centre de Recherches de Pau, SNPA, 5, 31-44.

Kontrovitz, M. 1975. A study of the differential transportion of Ostracodes. Journal of Paleontology, 49, 937-941.

Kontrovitz, M. 1980. Ostracode shells as potential indicators of thermal history of sediments. Geological Society of America Abstract with Program, 12, 67.

Kontrovitz, M. 1987. Ostracode shells as indicators of thermal history. Transactions of the Gulf Coast Association of Geology, 37, 383-392.

Kontrovitz, M. and Dehon, R.A. 1983. Diagenetic changes to microfossils: Experimental studies. Gulf Coast Association of Geological Societies Transactions, 31, 323-324.

Kontrovitz, M. and Nicolich, M.J. 1979. On the response of ostracode valves and carapaces to water currents. In: Taxonomy, bioestratigraphy and distribution of ostracodes (Ed. N. Krstic). Serbian Geological Society, Beograd, 269-272.

Kontrovitz, M. and Snyder, S.W. 1981. Reliability of microfossil assemblages as palaeoenvironmental indicator. Gulf Coast Association of Geological Societies Transactions, 31, 323-324.

Kontrovitz, M., Snyder, S.W. and Brown, R., 1978. A flume study the movement of foraminiferal test. Palaeogeography, Palaeoclimatology, Palaeoecology, 23, 141-150.

Kontrovitz, M., Dehon, R.A. and Myers, J. H. 1983. Simulated burial of ostracode shells: progressive alteration. In: Applications of Ostracoda (Ed. R.F. Maddocks). University of Houston Geoscience, 62-67.

Kontrovitz, M., Mann, J. B. and Barnett, S. D. 1988. Ostracode shell colour: a potential paleotetemperature indicator. Geological Society of America Abstract with Program, 20, 221.

Kontrovitz, M., Ainsworth, N.R., Burnett, D.B. and Slack, J.M. 1992. Indices color in ostracode shells: An experimental study. University of Kansas Paleontological Contributions Paper, 2, 1-10.

Kornicker, S. L. and Sohn, I. G. 1971. Viability of ostracode eggs egested by fish and effect of digestive fluids on ostracode shells ecologic and paleoecologic implications. In: Colloque sur la Paléoécologie des Ostracodes (Ed. H.J. Oertli). Bulletin du Centre de Recherches de Pau, SNPA, 5, 125-135.

Krumbein, W.C. and Sloss, L.L. 1963. Stratigraphy and Sedimentation. Freeman and Co., San Francisco, 660 pp. 
Langer, W. 1973. Zur Ultrastruktur, Mikromorphology und Taphonomy des Ostracoda-Carapax. Paleontographica A, 144, 54 pp.

Michelsen, O. 1975. Lower Jurassic bioestratigraphy and ostracods of the Danish Embayment. Danmarks Geologiske Undersogelse, 104 (2), 289 pp.

Oertli, H.J. 1971. The aspect of ostracoda faunas - A possible new tool in petroleum sedimentology. In: Colloque sur la Paléoécologie des Ostracodes (Ed. H.J. Oertli). Bulletin du Centre de Recherches de Pau, SNPA, 5, 137-151.

Parsons, K.M. and Brett, C.E. 1991. Taphonomic Processes and biases in modern marine environments. In: Fossilization: the processes of taphonomy (Ed. S.K. Donovan). Belheven Press, London, 22-65.

Pettijohn, F.J., Potter, P.N. and Sieber, R. 1972. Sand and Sandstone. Springer-Verlag, $618 \mathrm{pp}$.

Pokorny, V., 1971. The diversity of fossil ostracode communities as and indicator of Palaeogeographic conditions. In: Colloque sur la Paléoécologie des Ostracodes (Ed. H.J. Oertli). Bulletin du Centre de Recherches de Pau, SNPA, 5, 45-61.

Pokorny, V. 1978. Ostracodes. In: Introduction to Marine Micropaleontology (Eds. B.U. Haq and A. Boersma). Elsevier, Amsterdam, 4, 109-149.

Speyer, S.E. and Brett, C.E. 1991. Taphofacies Controls. Background and Episodic Processes in Fossil Assemblage Preservation. In: Taphonomy: Releasing the Data Locked in the Fossil Record (Eds. P.A. Allison and D.E.G. Briggs). Vol 9, Topics in Geobiology, Plenum Press, New York, 1, 501-545.

Whatley, R. 1983. The application of Ostracoda to Palaeoenvironmental Analysis. In: Applications of Ostracoda (Ed. R.F. Maddocks). University of Houston Geoscience, 51-77.

Whatley, R. 1988. Ostracoda and Palaeogeography. In: Ostracoda in the Earth Sciences (Eds. P. De Deckker, J.P. Colin and J.P. Peypouquet), 103-123. 